\title{
Commentary on De Souza and Lokan (2019)
}

\author{
Jan Miyake \\ Oberlin College and Conservatory [1]
}

\begin{abstract}
This contribution is a brief commentary on the paper "Hypermetrical Irregularity in Sonata Form: A Corpus Study" by Jonathan De Souza and David Lokan. The original paper explores the hypothesis that in a sonata form, the development is more hypermetrically stable than the exposition. The published data support the hypothesis. The commentary suggests other paths for discussion and further tweaks to the study that may better show how data support or contradict De Souza and Lokan's intuitions.
\end{abstract}

Submitted 2019 November 26; accepted 2019 December 2.

Published 2020 July 6; https://doi.org/10.18061/emr.v14i3-4.7568

KEYWORDS: Sonata form, hypermeter, commentary

DE Souza and Lokan explore the hypothesis that development sections are more hypermetrically stable than expositions. The methodology is clean and simple. The corpus consists of 60 works written by 10 composers with composition dates spanning 131 years (1761-1892). Haydn and Mozart are each represented by 10 symphonic sonata-form movements, and the other eight composers are represented by their complete symphonic output, presumably less than 10 movements. The data were collected by two independent raters working from a set of six rules: the four preference rules stated by De Souza and Lokan, a fifth to set quadruple hypermeter as the default, and a sixth to guide analyses of "ambiguous or transitional passages." The team measured two types of hypermetrical disruptions: deviations and shifts. Deviations are a count of non-four-bar hypermeasures. Shifts are a count of consecutive hypermeasures that change in size. The data were then normalized as a proportion of the total number of hypermeasures.

The results support the hypothesis. Over the entire corpus, De Souza and Lokan show that the proportion of hypermetrical shifts and hypermetrical deviations was lower in developments than expositions. The hypothesis still holds when the data is broken down by composer. Since hypermetrical deviations did not have a normal distribution, additional analysis was only provided about hypermetrical shifts. Of the further analysis, most interesting to me was the weak negative correlation between composition date and proportion of hypermetrical shifts.

One area of concern for me is the use of proportions in the normalization of the data. Given the wide timespan of the composition dates, I imagine the developments (and corresponding expositions) are of drastically varying lengths. A short Haydn development may have as few as 16 bars (Symphony No. 5, mvt. 4, written sometime between 1760 and 1762), which results in a maximum of four four-bar hypermeasures and only large fluctuations available in the proportion of deviations and shifts (e.g., $2 / 4=.5$, $3 / 4=.75$ ). At the other extreme, the almost 250 measures in the development section of Beethoven's Eroica could contain over 60 four-bar hypermeasures; these proportions have much smaller fluctuations (e.g., $2 / 60=.03,3 / 60=.05$ ). Works with short sections may have an overly large impact on the mean owing to the lack of nuance available in their proportions of hypermetrical disruptions to total hypermeasures.

De Souza and Lokan's discussion piqued my curiosity about related questions. For example, in our current analytical climate of rich sonata form discussions, I was curious about what kinds of sonata forms they included. Clearly, the authors included none that omit the development, but they also did not include Beethoven's entire symphonic sonata form output (as claimed). This output is more than 10 movements because Beethoven frequently used sonata form for movements other than the first movement. A second path of inquiry could be opened when considering slow-movement sonata forms. I suspect that approaches to the four-bar hypermeter differ in a slow inner movement. How many of the works in the corpus are not first movements? 
The authors forward three intriguing thoughts on why developments are more hypermetrically stable than expositions. First, since developments often hold sequences, and since sequences project a regular hypermeter, hypermetrical shifts and deviations are less common. Second, Ng (2012) and Temperly (2003) have shown that within expositions, the primary- and secondary-theme zones tend to be beginningaccented while the closing zone tends to be end-accented. These hypermetrical differences in the exposition presumably set the stage for a higher number of hypermetrical shifts and deviations. Third, a hypermetrically stable development provides a balance to other syntactic layers such as harmony and modulation that are less stable relative to the exposition.

Repeating the study while controlling for the length of the sections would allow for a more robust discussion of the role of sequences in the hypermeter of developments. This tweak would also address this issue of small sections that I discussed above. In their discussion, De Souza and Lokan return to the idea that developments might derive their relatively more stable hypermeter from the presence of sequences. However, it is less likely for sequences to determine a hypermeter, especially a four-bar hypermeter in a short development. Despite the fact that Caplin (1998, after Ratz, 1973) claims sequences are the core of the development, it could be insightful to know how many of the 60 works actually had sequences in the development sections. Is there a hypermetrical shift upon entering and exiting the sequences, or do they emerge from the pre-existing hypermeter? Could the lack of sequences help explain the hypermetrical instability of composers like Haydn? De Souza and Lokan have offered a solid launching point for larger studies that could help us better understand hypermeter and its relationship to sequences in development sections.

\section{ACKNOWLEDGEMENTS}

This article was copyedited by Tanushree Agrawal and layout edited by Diana Kayser.

\section{NOTES}

[1] Correspondence can be addressed to: Jan Miyake, Associate Professor of Music Theory, Oberlin College and Conservatory, Oberlin, OH 44074. Jan.Miyake@oberlin.edu.

\section{REFERENCES}

Caplin, W. E. (1998). Classical form: A theory of formal functions for the instrumental music of Haydn, Mozart, and Beethoven. New York: Oxford University Press.

Ng, S. (2012). Phrase rhythm as form in classical instrumental music. Music Theory Spectrum, 34, 51-77. https://doi.org/10.1525/mts.2012.34.1.51

Ratz, E. (1973) Einführung in die musikalische Formenlehre: Über Formprizipien in den Inventionen und Fugen J. S. Bachs und ihre Bedeutung für die Kompositionstechnik Beethovens. $3 \mathrm{~d}$ ed. Vienna: Universal.

Temperley, D. (2003). End-accented phrases: An analytical exploration. Journal of Music Theory, 47(1), 125-154. https://doi.org/10.1215/00222909-47-1-125 